\title{
Aqueous solution polymerization of acrylamide:synthesis and optimization
}

\author{
Haidi Cai ${ }^{1, a}$, Changhong $\mathrm{Li}^{2, \mathrm{~b}}$, Feng Zhao ${ }^{1, \mathrm{C}^{*}}$ \\ ${ }^{1}$ Jiangxi Key Laboratory of Organic Chemistry, Jiangxi Science \& Technology Normal University, \\ Fenglin Street Nanchang, jiangxi 330013, China. \\ ${ }^{2}$ Changjiu Agrochemical Co.,Ltd,Nanchang, jiangxi 330012,China.

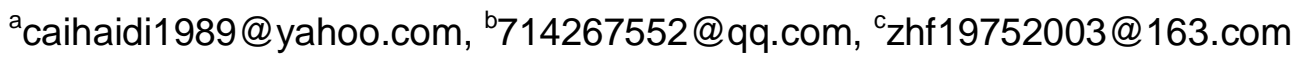

Keywords: Polyacrylamide, Aqueous solution polymerization,Composite initiator, Homopolymer Abstract. The polymerization of acrylamide initiated by 2,2'-Azobis(2-methylpropionamidine)dihydrochloride and ammonium persulfate was studied in aqueous solution at $6 \mathrm{C}$.The effect of variables such as acrylamide concentration and initiator concentration, additive concentration were investigated. When the concentration of acrylamide was $25 \%$, azo initiator concentration was $0.4 \%$,redox initiator concentration was $0.062 \%$,a very soluble acrylamide polymer was synthesized.

\section{Introduction}

Polyacrylamide is one of the most widely used varieties of water soluble polymer compound. It was widely applied to the fields such as chemical industry,medicine, water treatment and so on [1]. The main synthetic methods of polyacrylamide are aqueous solution polymerization, inverse emulsion polymerization method and the method of suspension polymerization method and so on[2-4]. The water solution polymerization method is low cost, and small damage to the environment .But there are still some problems along with the polyacrylamide powder products. Its low relative molecular mass and bad solubility make it unable to meet the demand of industry and environment, and the low dissolution rate leads to inconvenient application and storage[5]. Therefore, the research on the development of polyacrylamide with high molecular weight synthesized by aqueous exists great practical significance.

\section{Experimental}

Materials. Acrylamide (AM) was purchased from Changjiu Agrochemical Co.Ltd. (Jiangxi, China); Ammonium peroxydisulfate used as the oxidant was provided by Yongda (Tianjin, China);Ammonium iron(II) sulfate used as reducing agent was provided by Aladdin;2,2'-Azobis(2-methylpropionamidine)dihydrochloride was provided by Aladdin.

Experimental method. For this study, a series of steps were carried out as follows:

(1) The desired amount of acrylamide and other additive was weighed out and transferred to jars,

(2) The jar was sealed with nitrogen in addition to oxygen,

(3) A syringe was used to transfer the desired amount of initiator into the sealed jars containing the monomer.

(4) After the polymerization reaction was finished, the reaction jars was opened and the polymer was removed out into a beaker containing acetone then the mixture was standing for some time.The resulting products were dried and crushed.

\section{Results and Discussion}

Effect of acrylamide concentration. The choice of the acrylamide concentration plays a vital role in polymerizing acrylamide.This paper studies the effect of different concentration.It is shown in Fig.1. 


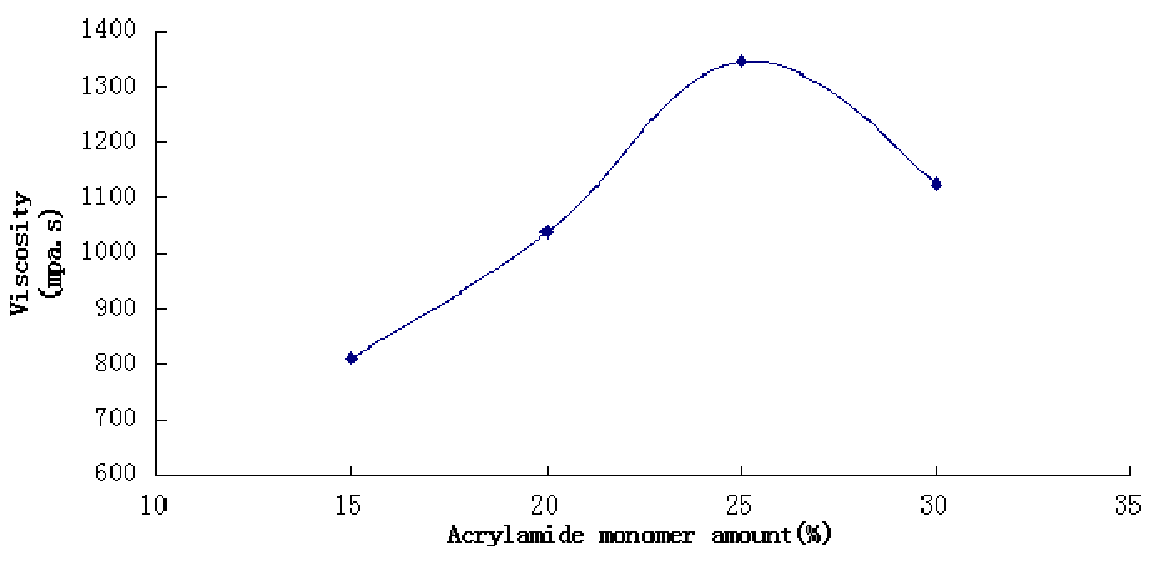

Fig. 1 The influences of different concentration content on polymer viscosity

The size of the viscosity has great relative to acrylamide monomers.. Polymerization viscosity increase with acrylamide concentration as a result of increase in the reaction constant. After reaching the maximum value, the polymerization viscosity was decreased with higher of acrylamide concentration. The polymer viscosity increased first and then decreased, as shown in Fig. 1, when the concentration of acrylamide was $25 \%$, the viscosity of the polymer was maximum.

Effect of azo initiators concentration. Azo initiators have a significant impact in acrylamide polymerization. The biggest advantage of azo initiators are sensitive to solvents and impurities, by introducing a polar group can increase its water solubility[6]. Azo initiator concentration on viscosity is shown in Fig.2.

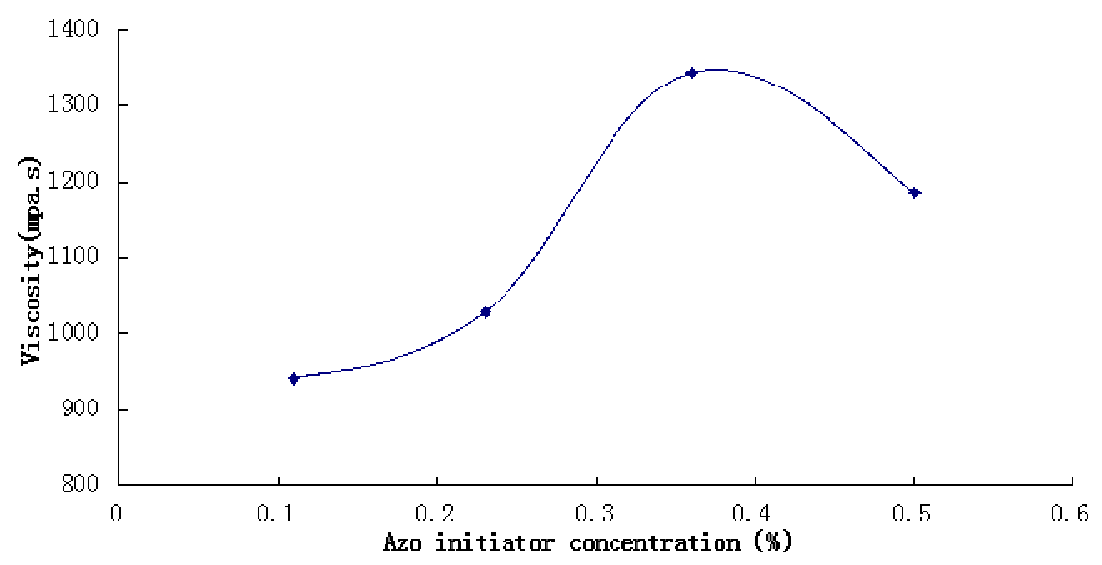

Fig.2 The influences of different Azo initiator on polymer viscosity

Experiments showed that different amounts of azo initiators impact on the viscosity. Polymerization viscosity increase with azo initiators concentration, After reaching the maximum value, the polymerization viscosity was decreased with higher of azo initiators concentration. The polymer viscosity increased first and then decreased,as shown in Fig.2., when the concentration of azo initiators was $0.4 \%$, the viscosity of the polymer was maximum.

Effect of different reddox concentration. Polymer viscosity with increasing initiator concentration increased first and then decreased. We investigate the effect of initiator concentration on polymerization of acrylamide.The viscosity of polymer for the reactions performed at redox initiator concentration is shown in Fig.3. 


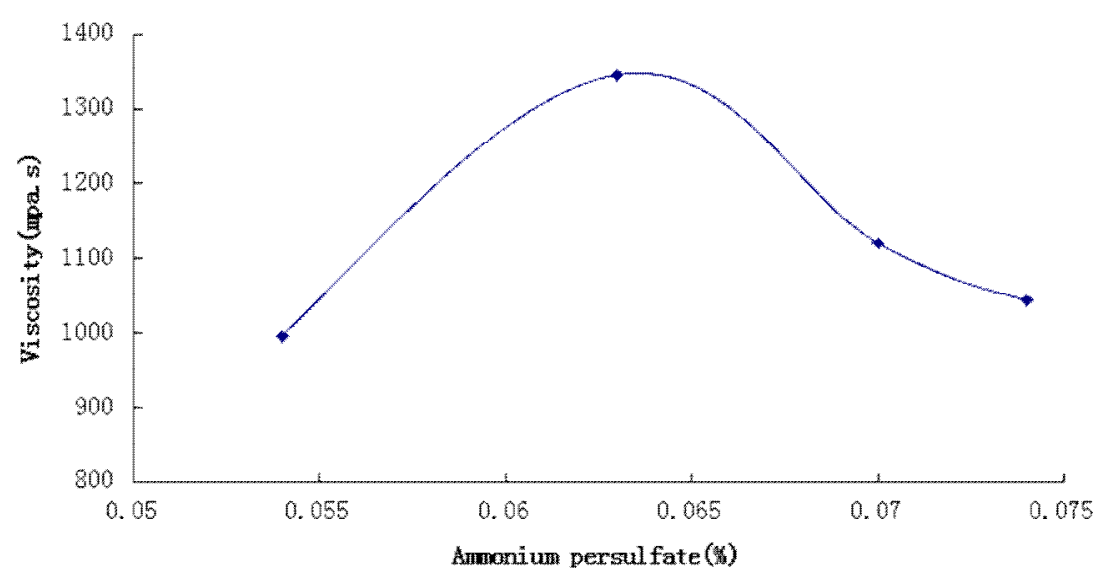

Fig.3 The influences of ammonium persulfate on polymerization viscosity

It is clear that, from Fig.3, polymerization viscosity are increased with the increased of initiator when the concentration is lower than $0.062 \%$, however it showed decreasing when the concentration higher than $0.062 \%$. The main reason of this phenomenon: when the concentration of oxidant is small, with the increase of initiator concentration that reactive intermediates increased and polymerization reaction completely. So the increase of the molecular weight of polymer and showing the polymerization viscosity increase. But when the initiator concentration exceeds a certain amount, activity center is more. So that the reaction speed is quick. Eventually leading to the heating rate is too large and the heat of reaction is not easy to spread out. The molecular chain is easy to break. The molecular weight of the polyacrylamide decreases, which leads to the polymerization viscosity reducing.

Effect of different additives. As we all know, to solve the solubility problem PAM, one way is to add a chain transfer agent in the polymerization system, the polymerization process to reduce the PAM branched chain line into crosslinked within PAM chains and chains of suppression. The effect of chain transfer agent sodium formate on polymer viscosity is shown in Fig.4.

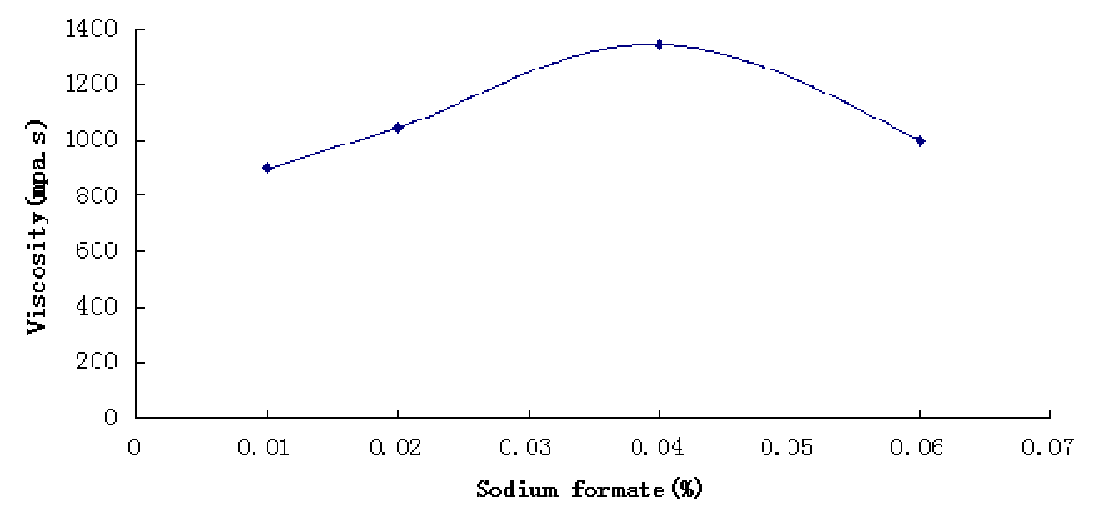

Fig.4 The effect of chain transfer agent sodium on polymer viscosity

It is clear that, from Fig.4, with increasing concentrations of sodium formate, the viscosity of polyacrylamide showed a decreasing trend after the first increase. When the amount is more than $0.04 \%$, it starts to sharply reduce the viscosity of the polymer. This is because during the polymerization reaction chain transfer effect, consumption of reactive free radicals, inhibit smooth progress of the polymerization chain reaction, the polymer molecular weight degradation. But adding an appropriate amount of sodium, can improve the solubility of the polymer, but also can be obtained higher molecular weight products, to meet the requirements of practical application. 


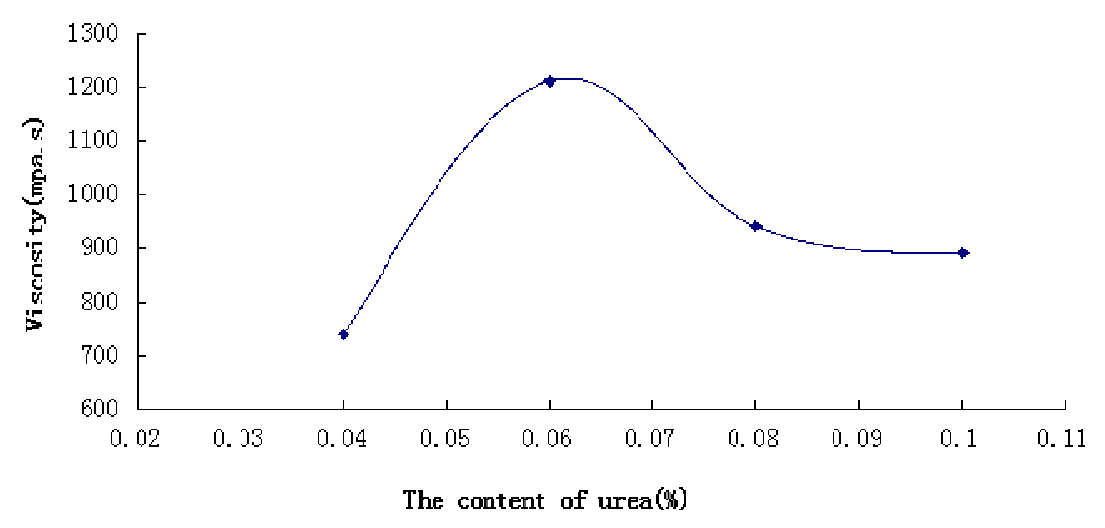

Fig.5 The effect of urea on polymer viscosity

The effect of urea on the viscosity of the polymer have been studied.It is shown in Fig.5.

It is clear that,from Fig.5, with the increase concentration of solubilizers, viscosity of the polymer after the first increases and then decreases. This is because when the concentration of increase is less solvent, the solubilizing agents can be used as an auxiliary reducing agent involved in redox processes conducive to growth kinetic chain length, thereby improving product molecular weight. With the increase of the concentration of the solubilizing agent, the polymer dissolution time is reduced. This is because the active solubilizing amide groups react with molecules in the amide group, can effectively prevent cross-linking in the polymer molecules to improve the water-soluble product. From the viscosity of the product to consider,solubilizers was selected for this purpose at the concentration of $0.06 \%$.

\section{Conclusions}

Aqueous solution polymerization technique was used to synthesis PAM by free radical homopolymer of water-soluble monomers. In this study,the optimum synthetic conditions can be achieved according to the concentration of acrylamide is $25 \%$, the best concentration of azo initiator concentration is $0.036 \%$, the best concentration of redox concentration is $0.062 \%$, the chain transfer agent concentration of $0.04 \%$, and the solubilizer concentration of $0.06 \%$.

\section{References}

[1] Hong-RuLin.Solution Polymerization of acrylamide using PotassiumPersulfate as aninitiator.kenitic studies, temperature, and $\mathrm{pH}$ dependence[J].EuroPean Polynler Joumal,2001,37:1507-1510.

[2] Hernandez-Barajas J, Hunkeler DJ (1997) Inverse-emulsion copolymerization of acrylamide and quaternary ammonium cationic monomers with block copolymeric surfactants: copolymer composition control using batch and semi-batch techniques. Polymer 38:449-458.

[3] Abdollahi Z, Frounchi M, Dadbin S (2011) Synthesis, characterization and comparison of PAM, cationic PDMC and P(AM-co-DMC) based on solution polymerization. J Ind Eng Chem.17:580-586.

[4] Wu YM, Wang CX, Xu J (2010) Aqueous dispersion polymerization of amphoteric polyacrylamide. J Appl Polym Sci 115:1131-1137.

[5] $\mathrm{Xu}$ zhang. The synthesis of high molecular weight polyacrylamide and evaluation of flocculation performance [D].Inner Mongolia University of Technology.2013:3-5.(In Chinese)

[6] F Daobing.polyacrylamide[M].Beijing: Chemical Industry Press, 2006.(In Chinese) 